\title{
Heterologous Expression of a Salinity and Developmentally Regulated Rice Cyclophilin Gene (OsCyp2) in E. coli and $S$. cerevisiae Confers Tolerance Towards Multiple Abiotic Stresses
}

\section{Sumita Kumari · Prabhjeet Singh •}

Sneh L. Singla-Pareek · Ashwani Pareek

Published online: 31 March 2009

(C) Humana Press 2009

Erratum to: Mol Biotechnol

DOI 10.1007/s12033-009-9153-0

Figures 3 and 4 in the original publication were improperly aligned. The Figs. 3 and 4 should appear as follows:

The online version of the original article can be found under doi:10.1007/s12033-009-9153-0.

S. Kumari · A. Pareek ( $\square)$

Stress Physiology and Molecular Biology Laboratory,

School of Life Sciences, Jawaharlal Nehru University,

New Delhi 110067, India

e-mail: ashwanip@mail.jnu.ac.in

\section{S. L. Singla-Pareek}

Plant Molecular Biology, International Centre for Genetic Engineering and Biotechnology, New Delhi 110067, India

P. Singh

Department of Biotechnology, Guru Nanak Dev University,

Amritsar 143005, India 


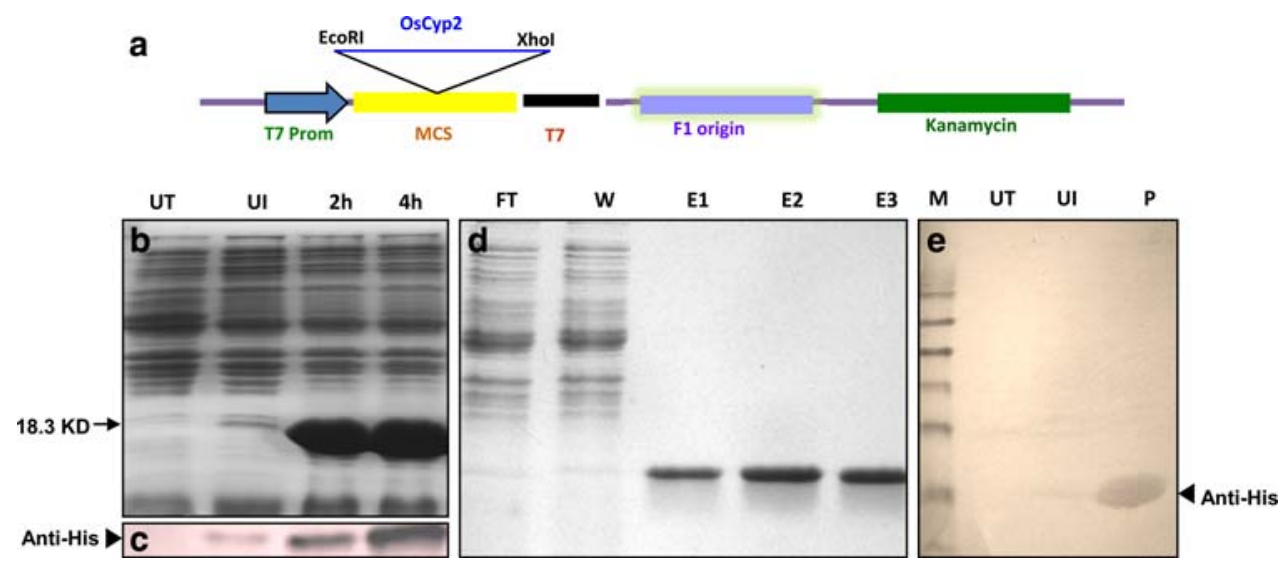

Fig. 3 a Schematic diagram of pET28aOsCyp2 construct used for His tagged recombinant protein expression. b Coomassie stained PAGE showing crude protein extract from untransformed (UT), uninduced (UI) and OsCyp 2 transformed BL21DE3 cells, an induced band corresponding to OsCyp2 after 2 and $4 \mathrm{~h}$ of IPTG induction is visualized (marked by arrow) and c the corresponding western analysis using anti-His antibodies (marked by arrow head). d
Coomassie stained PAGE showing Ni-NTA affinity purified recombinant OsCyp2; $F T$ flow through, $W$ wash fraction, E1: first eluate fraction, E2: second eluate fraction and E3: third eluate fraction and e the corresponding western analysis using anti-His antibodies (marked by arrow head); $M$ marker, UT untransformed, UI uninduced, $P$ purified protein
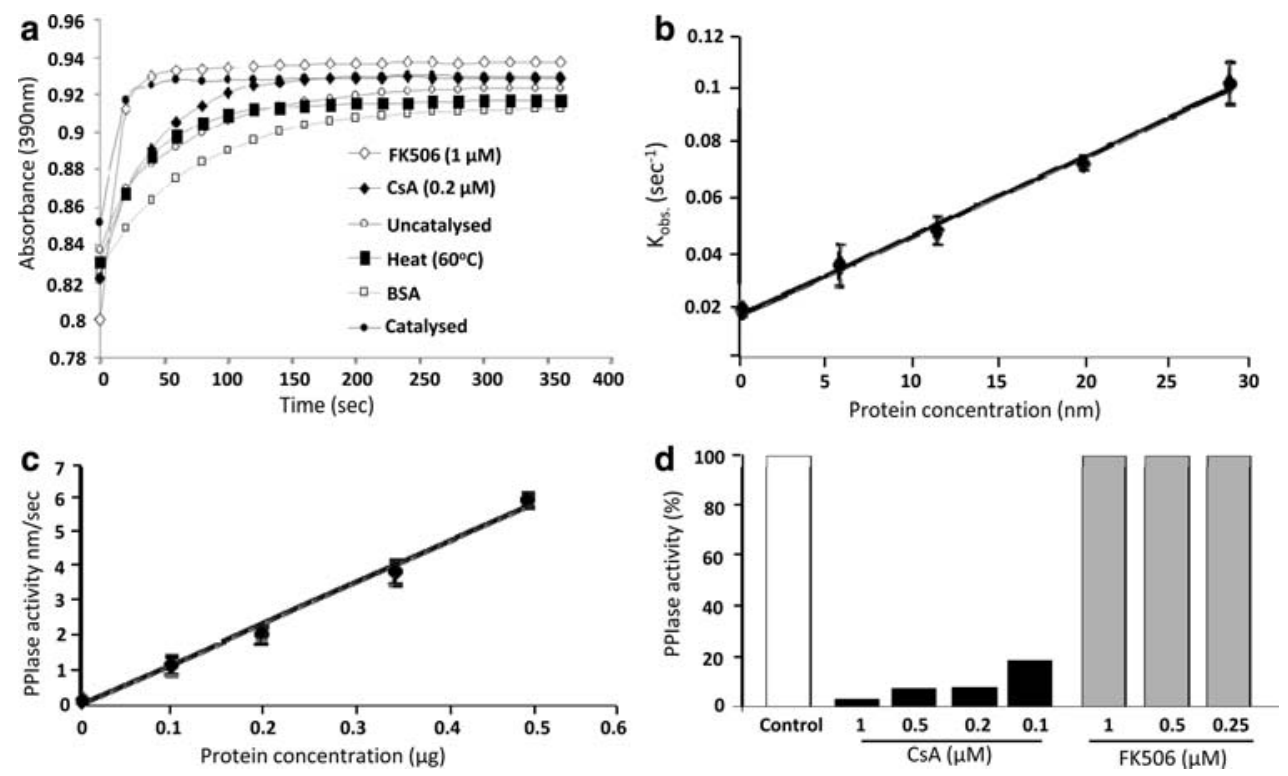

Fig. 4 a Kinetics of the hydrolysis of $N$-succinyl-ala-ala-pro-phe- $p$ nitroanilidine followed in the presence ( filled circle) and absence of purified recombinant OsCyp2 $(\bigcirc$ empty circle).To study the effect of inhibitors the reaction was monitored in the presence of OsCyp2 along with PPIase inhibitors $(\diamond$ filled rhombus) CsA and $(\diamond$ empty rhombus) FK506. For thermostability reaction was performed in the presence of heat denatured $\left(60^{\circ} \mathrm{C}\right.$ for $10 \mathrm{~min}$ ) OsCyp2 ( filled

square). BSA was used as a negative control ( $\square$ empty square). Dependence of the rate constant (b), and the corresponding PPIase activity (c) as a function of protein concentration. d Dependence of the PPIase activity in fraction 100 with the addition of increasing concentrations of cyclophilin inhibitor CsA and FKBP inhibitor FK506 